\title{
Radiation pattern of the isolated pulsar PSR B1828-11
}

\author{
V. Rezania ${ }^{\star \star \star \star}$ \\ Institute for Advanced Studies in Basic Sciences, Gava Zang, Zanjan 45195, Iran \\ Received 9 September 2002 / Accepted 25 November 2002

\begin{abstract}
Based on the free precession model of the isolated pulsar PSR B1828-11, Link \& Epstein (2001) showed that the observed pulse durations require the radio beam to have a non-standard shape: the beam duration is larger for beam sweeps farthest from the dipole axis. In their analysis they assumed that the actual precession period is $\simeq 500 \mathrm{~d}$. Recent theoretical studies suggested that the actual precession period might be $\simeq 1000 \mathrm{~d}$ as seen in observations (Rezania 2003; Wasserman 2002). In this paper, in a good agreement with the observed data (Stairs et al. 2000), we model the changes of the pulse shape in a precession cycle with period $\simeq 1000 \mathrm{~d}$ and find that the variation of the pulse duration follows from a standard beam pattern in each cycle.
\end{abstract}

Key words. pulsar: individual: PSR B1828-11 - stars: neutron

\section{Introduction}

Analysis of the long-term observation of the spin behavior of the isolated pulsar PSR B1828-11 reveals periodic variations both in the pulse shape and the slow-down rate of the pulsar and shows strong Fourier power at periods of $\simeq 1000,500,250$, and $167 \mathrm{~d}$ (Stairs et al. 2000). Close correlations of the periodic changes in the pulse shape and duration with variations in the spin-down rate of the pulsar strongly suggest that the star's spin axis is freely precessing around the star's symmetry axis.

Recently Link \& Epstein (2001) studied the behavior of the observed pulse duration of PSR B1828-11 based on the free precession model. Since both $500 \mathrm{~d}$ and $250 \mathrm{~d}$ Fourier components have dominant contributions in the observed variations of period residual $\Delta p$, its derivative $\Delta \dot{p}$, and pulse shape of the beam, they suggested that the actual free precession period of the star is close to the strongest Fourier component $\simeq 500 \mathrm{~d}$. Then a coupling of nearly orthogonal magnetic dipole moment to the star's spin axis would provide the observed harmonic at period $250 \mathrm{~d}$. On this basis, they modeled the pulse duration variations as a function of beam's sweep angle $\Delta \Theta$ (see below for definition), and found that the beam pattern of radiations must be non-standard: the beam duration is larger for beam sweeps farthest from the dipole axis. As a result, both upper and lower parts of the emission region are wider than its middle, see Fig. 2.

As they mentioned this is required by the precession interpretation of PSR B1828-11.

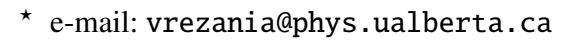

$\star \star$ Present address: Department of Physics, University of Alberta, Edmonton AB, Canada T6G 2J1.
Recent theoretical investigations on the free precessing motion of PSR B1828-11 provide new explanations for the reported data with the fundamental period $\sim 1000 \mathrm{~d}$, which was originally suggested by Jones \& Andersson (2001). Rezania (2003) considered the case in which the magnetic field of the star varies with time while the star is precessing. Then he found a condition under which a coupling of the star's crust with the time-varying magnetic radiation torque would produce the whole observed Fourier spectrum consistently. In a good agreement with the data he found that the fundamental precession period would be $\simeq 1000 \mathrm{~d}$. Alternatively, Wasserman (2002) showed that in general an oblique rotator must precess. By analysis of the mechanical energy of the system, he found that the minimum energy state for such star is a state where the star precesses. For strong magnetic stresses in the star's type II superconductor core he estimated the precession period as $P_{\mathrm{pre}} \simeq 2460 \mathrm{~d} /\left(\beta \cos \chi B_{12} H_{15}\right)$, where $B_{12}=B / 10^{12} \mathrm{G}$ is the star's magnetic field strength, $H_{15}=H / 10^{15} \mathrm{G}$ corresponds to the first critical field strength in a type II superconductor $\left(H_{\mathrm{cr}} \sim 10^{15} \mathrm{G}\right), \beta \sim 1$ and $\chi$ is the inclination angle of magnetic symmetry axis. For the case PSR B1828-11 with $B \sim 5 \times 10^{12} \mathrm{G}$, the precession period will be $P_{\text {pre }} \sim 1000 \mathrm{~d}$, if the inclination angle is $\chi \simeq 60^{\circ}$.

In this paper we study the pulse shape of PSR B1828-11 by assuming that the actual precession period of the star is $\simeq 1000 \mathrm{~d}$ rather than $\simeq 500 \mathrm{~d}$. We note that Link \& Epstein's calculations give the right behavior of the pulse shape variations provided the actual precession period is close to $\simeq 500 \mathrm{~d}$ only. For the case $P_{\text {pre }} \simeq 1000 \mathrm{~d}$, their analysis gives an incorrect prediction for the observed shape variations. Here, we generalize link \& Epstein's analysis and show that, for $P_{\text {pre }} \simeq 1000 \mathrm{~d}$, 


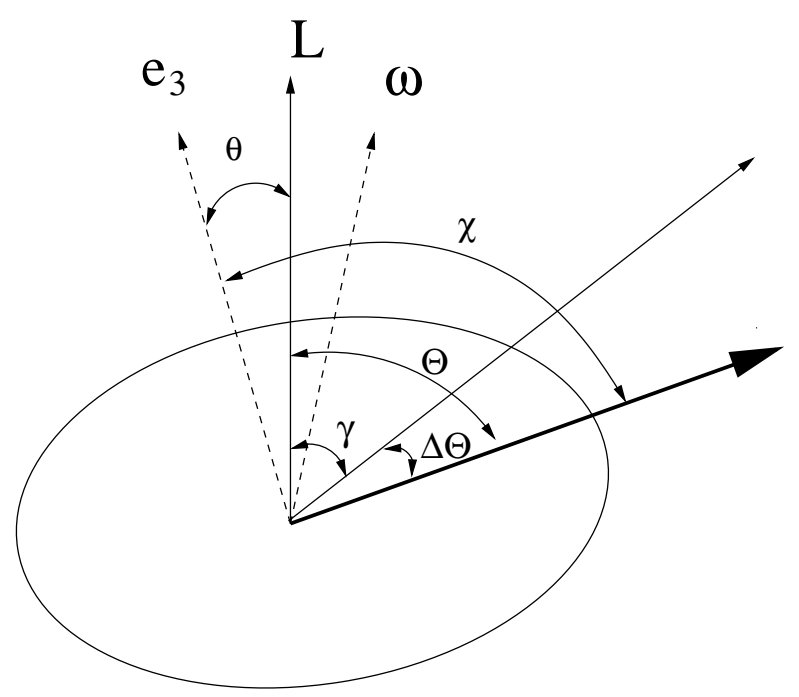

Fig. 1. Observing geometry. The angles defined at the instant the dipole moment $\boldsymbol{m}$ is in the plane containing the angular momentum $\boldsymbol{L}$ and the observer.

to get the correct behavior for the shape variations (compared with data) the radio beam of PSR B1828-11 must have the standard pattern.

\section{The model}

Let $x, y$, and $z$ represent an inertial coordinate system $\mathcal{S}$, and an observer is in the $x-z$ plane with $x>0$ and $z>0$. Now consider a rigid, biaxial rotating star with angular velocity $\omega$, the principal axes $\boldsymbol{e}_{1}, \boldsymbol{e}_{2}, \boldsymbol{e}_{3}$, and corresponding principal moment of inertia $I_{1}=I_{2} \neq I_{3}$. The star's angular momentum $\boldsymbol{L}$, along the $z$-axis in $\mathcal{S}$, is misaligned to the star's symmetry axis $\boldsymbol{e}_{3}$ by a wobble angle $\theta$, i.e. $\boldsymbol{L} \cdot \boldsymbol{e}_{3}=L \cos \theta$, see Fig. 1 . To study the pulse shape variations we look at the variation of the polar angle of the beam with respect to $\boldsymbol{L}$ (fixed in $\mathcal{S}$ ) that is equal to the polar angle of the magnetic dipole moment $\boldsymbol{m}^{1}$. The azimuthal and polar angles of the magnetic dipole moment $\boldsymbol{m}, \Phi$ and $\Theta$ in the inertial frame $\mathcal{S}$ are given by $\tan \Phi=m_{y} / m_{x}$ and $\cos \Theta=m_{z} / m=\sin \theta \sin \psi \sin \chi+\cos \theta \cos \chi$, where $\theta$ is the wobble angle, $\psi=\tan ^{-1} \omega_{1} / \omega_{2}=\pi / 2-\omega_{\mathrm{p}} t-\beta$ $\left(\omega_{i}=\boldsymbol{e}_{i} \cdot \omega\right)$ and $\chi$ is the inclination of magnetic field symmetry axis from the star's symmetry axis $\boldsymbol{e}_{3}$, i.e. $\boldsymbol{m} \cdot \boldsymbol{e}_{3}=m \cos \chi$. Here $\omega_{\mathrm{p}}=2 \pi / P_{\text {pre }}$ is free precession frequency and $\beta$ is a constant phase. Expanding $\cos \Theta$ to the first order in $\theta$ one finds $\Theta=\chi-\theta \cos \left(\omega_{\mathrm{p}} t+\beta\right)$. The latter shows the beam polar angle $\Theta$ changes sinusoidally about $\chi$, as the star precesses.

Now let $\gamma \equiv \xi+\chi$ be the polar angle of the observer in $\mathcal{S}$ (the constant angle between the observer and the angular momentum vector), see Fig. 1. Following Link \& Epstein (2001)

\footnotetext{
${ }^{1}$ Note that though the pulsar beam's direction is not necessarily in the same direction as the dipole moment $\boldsymbol{m}$, for simplicity Link \& Epstein (2001) defined a pulse as occurring when the azimuthal angle $\Phi$ of the magnetic dipole equals to the azimuth of the observer. As a result, $\dot{\Phi}$ is the observed pulse frequency. To compare our concluding result with one obtained by Link \& Epstein (2001), we use the same definitions.
}

A

B

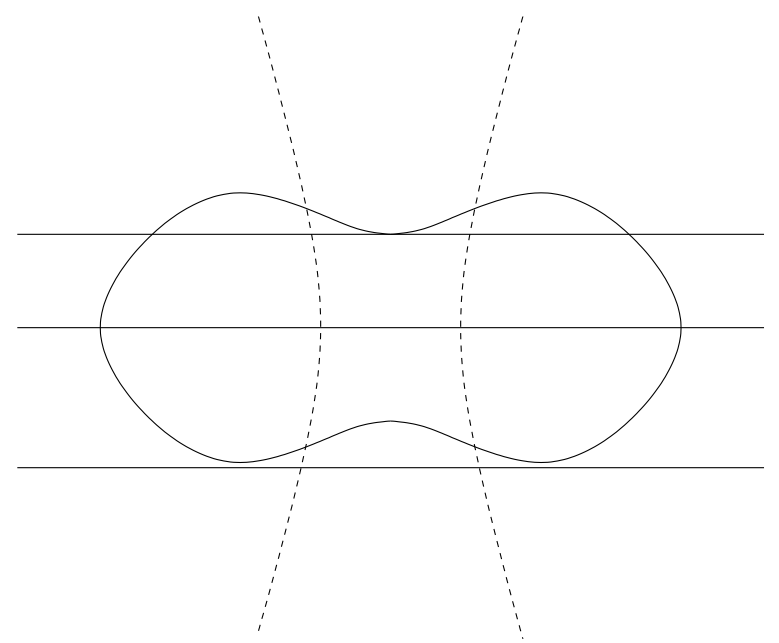

Fig. 2. The beam pattern for the radio emission from PSR B1828-11. The dashed parabolas represent the beam pattern proposed by Link $\&$ Epstein (2001) with $P_{\text {pre }} \simeq 500 \mathrm{~d}$, while the solid closed curve correspond to the $1000 \mathrm{~d}$ precession period. The radiation pattern is very close to multipole radiation pattern with $(\ell, m)=(1, \pm 1)$. The lines $\mathrm{B}, \mathrm{A}, \mathrm{C}$ represent the viewing angle at which the beam is the narrowest $(S \simeq 1)$, wider $(S \simeq 0.4)$, and widest $(S \simeq 0)$, respectively, for Link \& Epstein's model, while correspond to the widest $(S \simeq 0)$, narrower $(S \simeq 0.4$ ), and narrowest $(S \simeq 1$ ), respectively, for the model discussed here.

we define the sweep angle $\Delta \Theta$ as the difference in polar angle of observer, $\gamma$, and the dipole, $\Theta$ at the time of the pulse:

$\Delta \Theta \equiv \gamma-\Theta=\xi+\theta \cos \left(\omega_{\mathrm{p}} t+\beta\right)+\mathcal{O}\left(\theta^{2}\right)$.

Here $\xi$ is a free parameter which will be fixed later by fitting the data. Link \& Epstein assumed that the pulse duration $w$ is a function of $\Delta \Theta$ only, and has an extremum at $\Delta \Theta=0$. Then they expanded the pulse duration $w$ in terms of $\Delta \Theta$ as $w=w_{0}+w_{2}(\Delta \Theta)^{2}$. As it is clear, the latter expression would provide both $P_{\text {pre }}$ and $P_{\text {pre }} / 2$ components (due to the $\cos \left(\omega_{\mathrm{p}} t+\beta\right)$ and $\cos ^{2}\left(\omega_{\mathrm{p}} t+\beta\right)$ terms $)$ in the pulse duration. As a result, it gives the correct behavior of the observed pulse duration for $P_{\text {pre }} \simeq 500 \mathrm{~d}$. But with $P_{\text {pre }} \simeq 1000 \mathrm{~d}$, the $250 \mathrm{~d}$ Fourier component is missing. To get the correct behavior for the pulse shape variations during a $1000 \mathrm{~d}$ precession cycle, we take a more general expansion rather than Link \& Epstein (2001) as

$w=w_{0}+w_{2}(\Delta \Theta)^{2}+w_{4}(\Delta \Theta)^{4}$.

It is interesting to note that Eq. (2) provides the contribution of $P_{\text {pre }}, P_{\text {pre }} / 2, P_{\text {pre }} / 3$, and $P_{\text {pre }} / 4$ terms in the pulse duration. So with $P_{\text {pre }} \simeq 1000 \mathrm{~d}$, one would expect to observe $500 \mathrm{~d}$, $333 \mathrm{~d}$, and $250 \mathrm{~d}$ Fourier components in the pulse shape parameter. As reported by Stairs et al. (2000), the $1000 \mathrm{~d}$ and $333 \mathrm{~d}$ components though small (in comparing with $500 \mathrm{~d}$ and $250 \mathrm{~d}$ components) they have non-zero amplitude in the observed pulse shape parameter. These terms are missing in the Link \& Epstein's model.

Without loss of generality we assume that $w_{4}=1$. Following Stairs et al. (2000), we define the shape parameter as 
$S=\frac{A_{\mathrm{N}}}{A_{\mathrm{N}}+A_{\mathrm{W}}}$ where $A_{\mathrm{N}}$ and $A_{\mathrm{W}}$ are the fitted heights of the narrower and wider standard profiles respectively, so that $S \simeq 0$ for the wide pulses and $S \simeq 1$ for narrow ones. As a result, one can relate the shape parameter $S$ to the pulse duration $w$ of the observed beam by

$w=\max (w)(1-S)+\min (w) S$,

where $\max (w)$ and $\min (w)$ are the maximum and minimum values of the beam duration in the precession cycle. Combining Eqs. (2) and (3), one finds for $w_{2}>0$

$S=1-\frac{w_{2}+(\Delta \Theta)^{2}}{w_{2}+(|\xi|+\theta)^{2}}\left(\frac{\Delta \Theta}{|\xi|+\theta}\right)^{2}$,

while for $w_{2}<0$ we have

$S / S_{0}= \begin{cases}\frac{\left|w_{2}\right|-(\Delta \Theta)^{2}}{\left|w_{2}\right|-(|\xi|+\theta)^{2}}\left(\frac{\Delta \Theta}{|\xi|+\theta}\right)^{2} & \text { if }\left|w_{2}\right|>(|\xi|+\theta)^{2}, \\ 1-\frac{\left|w_{2}\right|-(\Delta \Theta)^{2}}{\left|w_{2}\right|-(|\xi|+\theta)^{2}}\left(\frac{\Delta \Theta}{|\xi|+\theta}\right)^{2} & \text { if }\left|w_{2}\right|<(|\xi|+\theta)^{2},\end{cases}$

where $S_{0}$ is a normalization factor. It is clear that the shape parameter depends on $\omega_{\mathrm{p}}, \beta, \theta, \xi$, and both the sign and magnitude of $w_{2}$. With the given values of $\theta \simeq 3^{\circ}$ and $P_{\text {pre }} \simeq 1000 \mathrm{~d}$ the shape parameter is determined by $\xi, w_{2}$, and $\operatorname{sign}\left(w_{2}\right)$ completely. In a good agreement with data, we find that $w_{2}$ must be negative with magnitude larger but close to $\left|w_{2}\right| \geq(|\xi|+\theta)^{2}$. Inserting the latter results in Eq. (2), one can easily show that the pulse duration $w$ is bigger when the beam sweeps closer to the dipole axis $(\Delta \Theta=0)$ rather than when it sweeps farther $(\Delta \Theta \neq 0)$. This means the beam pattern is standard: the beam duration is smaller for beam sweeps farthest from the dipole axis. Our result is completely in contrast with the result of Link \& Epstein (2001) who found that a non-standard beam pattern (the beam duration is larger for beam sweeps farthest from the dipole axis) is required to explain the data. In Fig. 2 we sample the pulse profile for different observer's viewing angles (the solid closed curve) and compare with the one (the dashed parabola) obtained by Link \& Epstein (2001). We find the radiation pattern of PSR B1828-11 is very close to multipole field radiation pattern with $(\ell, m)=(1, \pm 1)$ where $\ell$ and $m$ are orbital and azimuthal numbers. As the star precesses, the observer sees different sweeps corresponded to different viewing angles. Line B corresponds to the viewing angle (closest to the dipole axis) for which pulse is the widest, $S \simeq 0$ (narrowest, $S \simeq 1$ ) provided the star precesses with precession period $P_{\text {pre }} \simeq 1000 \mathrm{~d}\left(P_{\text {pre }} \simeq 500 \mathrm{~d}\right)$. Accordingly, the pulse profile is narrower, $S \simeq 0.4$ (wider, $S \simeq 0.4$ ) for line A and narrowest, $S \simeq 1$ (widest, $S \simeq 0$ ) for line $\mathrm{C}$.

By choosing an appropriate values for $\left|w_{2}\right|$ and $\xi$ one can easily fit the data. We found an acceptable fit on the pulse shape data of PSR B1828-11 by choosing $\left|w_{2}\right| \simeq 0.0034$ and $\xi=$ -0.01 for the given values of $\theta=3.2$ and $\omega_{\mathrm{p}}=2 \pi / 1016 \mathrm{~d}^{-1}$, see Fig. 3. Here the value of $(|\xi|+\theta)^{2} \simeq 0.0031$. We note that our fit curve which is calculated for free precession period $P_{\text {pre }}=$ $1016 \mathrm{~d}$, is indistinguishable from the one that was calculated by Link \& Epstein (2001) with free precession period $P_{\text {pre }}=$ $511 \mathrm{~d}$.

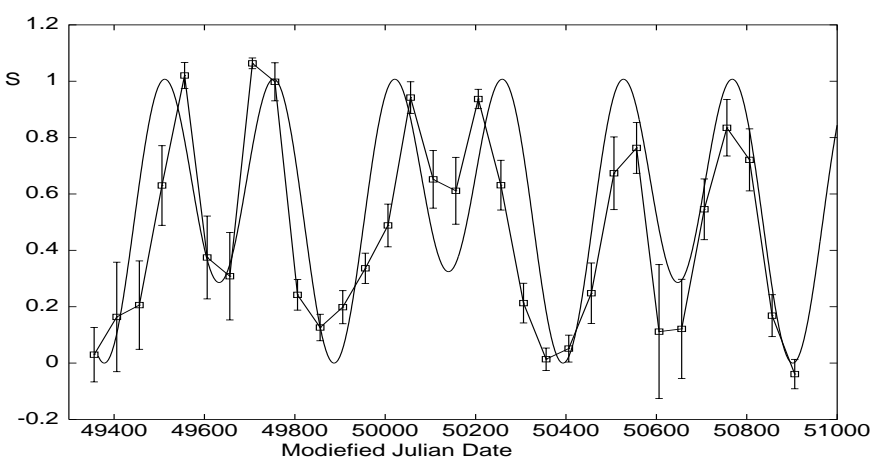

Fig. 3. The pulse shape parameter data $S$ for PSR B1828-11 (from Stairs et al. 2000). The shape parameter is defined as $S=\frac{A_{N}}{A_{N}+A_{W}}$ where $A_{\mathrm{N}}$ and $A_{\mathrm{W}}$ are the fitted heights of the narrower and wider standard profiles respectively, so that $S \simeq 1$ for the narrowest pulses and $S \simeq 0$ for wider ones. The data points are obtained by averaging $S$ over multiple bins, and the solid curve is the fit calculated with $P_{\text {pre }}=1016 \mathrm{~d}$, see text.

\section{Summary}

Previous study on the pulse shape variations of the isolated pulsar PSR 1828-11 by Link \& Epstein (2001) is based on the free precessing star with precession period $\sim 500 \mathrm{~d}$. Recent theoretical studies (Rezania 2003; Wasserman 2002) on the free precession of PSR B1828-11 suggested that the actual precession period might be $\simeq 1000 \mathrm{~d}$ as seen in observations. In this paper, by assuming that the actual free precession period of the isolated pulsar PSR B1828-11 is $P_{\text {pre }} \simeq 1000 \mathrm{~d}$ we studied the star's pulse shape variations during a precession cycle. To get the correct behavior in the pulse shape variations in comparing with the observed data, we expanded the pulse duration $w$ to forth order of $\Delta \Theta$ (the difference between polar angles of the observer and dipole axis at the time of the pulse). The forth order term is not considered by Link \& Epstein (2001). We found that the pulse duration $w$ is larger when the beam sweeps closer to the dipole axis $(\Delta \Theta=0)$ rather than when it sweeps farther to the dipole axis $(\Delta \Theta \neq 0)$, see Fig. 2 . This means that the variation of the pulse duration follows from a standard beam pattern in each cycle. Our result is in contrast with the result of Link \& Epstein (2001) who found that a non-standard beam pattern is required to explain the data.

Further, our model provides the contribution other Fourier harmonics, $1000 \mathrm{~d}$ and $333 \mathrm{~d}$, in the pulse shape parameter as seen in data. Although these terms have smaller (but nonzero) amplitudes rather than $500 \mathrm{~d}$ and $250 \mathrm{~d}$ Fourier components, they are forbidden in the Link \& Epstein's model. In addition, by taking into account a term $(\Delta \Theta)^{6}$ in Eq. (2), one can explain the $167 \mathrm{~d}$ Fourier component seen in data of PSR B1828-11 (Stairs et al. 2000). The latter which corresponds to $P_{\text {pre }} / 6$ Fourier component for $P_{\text {pre }} \simeq 1000 \mathrm{~d}$ is also forbidden for $P_{\text {pre }} \simeq 500 \mathrm{~d}$ free precession model.

Finally, we note that new observations of the pulse shape variations of PSR B1828-11 would be able to determine its radiation pattern. As predicted by Link \& Epstein (2001), in a $511 \mathrm{~d}$ precession cycle the observer would see both the broad upper and lower parts of the radio beam. This would 
be a critical observational evidence for the proposed theoretical This research was supported by the Natural Sciences and Engineering models, since by assuming $P_{\text {pre }} \simeq 1000 \mathrm{~d}$ as the fundamental Research Council of Canada.

precession period, the middle of the beam will be broader.

\section{References}

Acknowledgements. I would like to thank S. M. Morsink and S. Jones, D. I., \& Andersson, N. 2001, MNRAS, 324,811

Sengupta for their careful reading the manuscript and useful discus- Link, B., \& Epstein, R. I. 2001, ApJ, 556, 392

sions. The author is also grateful to I. Stairs for useful discussions and providing the timing data for PSR B1828-11. I would like to thank Yousef Sobouti and Roy Maartens for continuing encouragement.

Rezania, V. 2003, A\&A, 399, 653

Stairs, I. H., Lyne, A. G., \& Shemar, S. L. 2000, Nature, 406, 484

Wasserman, I. 2002, preprint [astro-ph/0208378] 